\title{
Hot Stuff for One Year (HSOY)
}

\section{A 583 million star proper motion catalogue derived from Gaia DR1 and PPMXL}

\author{
M. Altmann ${ }^{1}$, S. Roeser ${ }^{1,2}$, M. Demleitner ${ }^{1}$, U. Bastian ${ }^{1}$, and E. Schilbach ${ }^{1,2}$ \\ 1 Zentrum für Astronomie der Universität Heidelberg, Astronomisches Rechen-Institut, Mönchhofstr. 12-14, 69120 Heidelberg, \\ Germany \\ e-mail: maltmann@ari.uni-heidelberg.de \\ 2 Zentrum für Astronomie der Universität Heidelberg, Landessternwarte Königsstuhl, Königsstuhl 12, 69117 Heidelberg, Germany
}

Received 4 January 2017 / Accepted 10 February 2017

\begin{abstract}
Context. Recently, the first installment of data from the ESA Gaia astrometric satellite mission (Gaia DR1) was released, containing positions of more than 1 billion stars with unprecedented precision. This release contains the proper motions and parallaxes, however, for only a subset of 2 million objects. The second release will include those quantities for most objects.

Aims. In order to provide a dataset that bridges the time gap between the Gaia DR1 and Gaia DR2 releases and partly remedies the lack of proper motions in the former, Hot Stuff for One Year (HSOY) was created as a hybrid catalogue between Gaia and ground-based astrometry. This catalogue features proper motions (but no parallaxes) for a large percentage of the DR1 objects. While not attempting to compete with future Gaia releases in terms of data quality or number of objects, the aim of HSOY is to provide improved proper motions partly based on Gaia data and to allow studies to be carried out now or as pilot studies for later projects requiring higher precision data.

Methods. The HSOY catalogue was compiled using the positions taken from Gaia DR1 combined with the input data from the PPMXL catalogue, employing the same weighted least-squares technique that was used to assemble the PPMXL catalogue itself. Results. This effort resulted in a four-parameter astrometric catalogue containing 583 million stars with Gaia DR1 quality positions and proper motions with precisions from far less than 1 mas/yr to 5 mas/yr, depending on object brightness and location on the sky.
\end{abstract}

Key words. astrometry - catalogs - Galaxy: kinematics and dynamics - proper motions

\section{Introduction}

Gaia, the 1 billion star astrometric space mission by ESA (Gaia Collaboration 2016a), set out in late 2013 to revolutionise (among many other fields of astronomy) our understanding of the kinematics, structure, and evolution of our Galaxy. On September 14, 2016, the Gaia consortium published the first Gaia Data release, Gaia DR1 (Gaia Collaboration 2016b), containing the positions and broadband photometry for 1.143 billion objects. Given the short time span of the measurements incorporated into this release (less than 15 months), the separation of parallaxes and proper motions was not possible. Therefore Gaia DR1 in general does not include these quantities with the exception of a small subset of those 2 million stars already present in the HIPPARCOS (ESA 1997) or Tycho 2 catalogues (Høg et al. 2000). Using this data as a first epoch, proper motions and parallaxes could be disentangled, yielding the Tycho Gaia Astrometric Solution (TGAS; Michalik et al. 2015). Thus most astrometry-related projects within the astronomical community might focus on the TGAS data and on preparing for the next Gaia release.

In the meantime, we present a very short-lived yet powerful astrometric catalogue, adding value and scientific use cases to Gaia DR1. It provides proper motions for 583 million objects,

\footnotetext{
* The HSOY catalogue is only available at the CDS via anonymous ftp to cdsarc.u-strasbg. fr (130.79.128.5) or via http://cdsarc.u-strasbg.fr/viz-bin/qcat?I/339
}

i.e. for more than half of the objects for which Gaia DR1 only gives positions. This is achieved by combining data from the PPMXL catalogue (Roeser et al. 2010) and Gaia DR1 positions. Named Hot Stuff for One Year (HSOY), highlighting its shortlived nature, we intend to partly fill the gap in time between the ultra-precise positions of DR1 and the ultra-precise full five parameter astrometry of DR2.

Until HSOY is superseded by Gaia DR2, this catalogue presents the best set of proper motions in existence in the magnitude range fainter than TGAS to $G=20 \mathrm{mag}$ and is a valuable base for studies of stellar kinematics.

Section 2 describes the assembly of this catalogue and its input data and gives the overall characteristics of this catalogue. In Sect. 3 we demonstrate the improvement of the precision of proper motions of HSOY with respect to current entirely groundbased values with two science case examples.

\section{Presenting HSOY}

\subsection{Construction and stellar content of HSOY}

The HSOY catalogue was constructed with the method previously used to assemble the PPMXL catalogue (Roeser et al. 2010), which itself now forms one of the input datasets for HSOY. The input data for PPMXL were the 2MASS (Skrutskie et al. 2006) and USNO-B1.0 catalogues (Monet et al. 2003), and the input data for HSOY were PPMXL and 


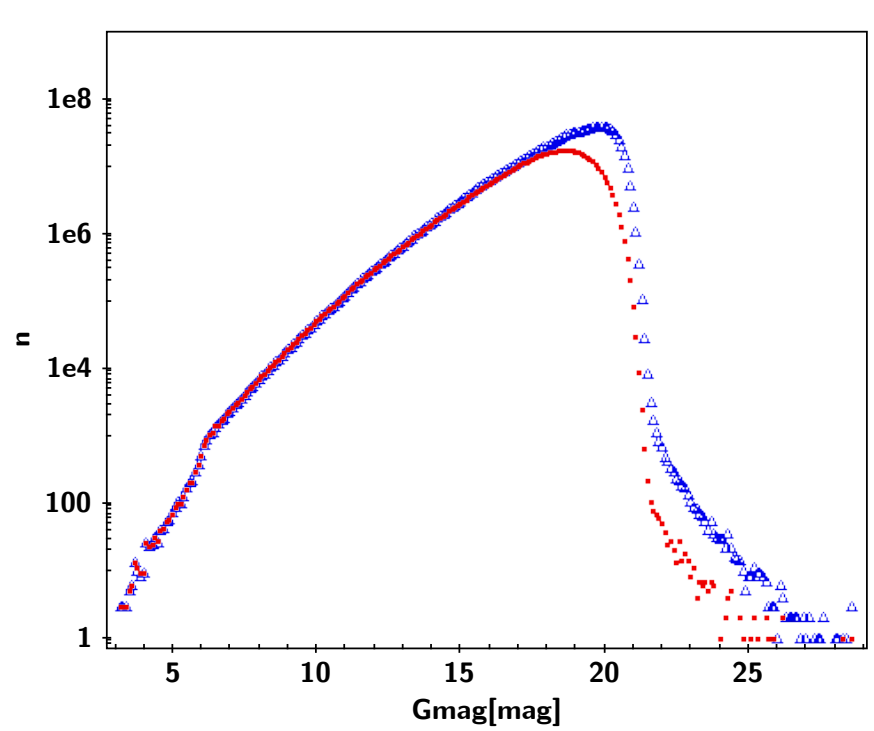

Fig. 1. Distribution of object counts in HSOY over the Gaia $G$ magnitude (red filled circles). As comparison, the object counts for Gaia DR1 are also shown (blue open triangles).

Gaia DR1. The procedure of construction, described in detail in Roeser et al. (2010), involves cross matches between the datasets and a weighted least-squares fit to derive positions and proper motions.

The PPMXL catalogue contains about 900 million and Gaia DR1 includes 1.1 billion sources. Yet HSOY only contains 583001653 entries, i.e. about $50-60 \%$ of the object numbers of the input catalogues. Figure 1 shows the object counts for both HSOY and Gaia DR1. Of course, HSOY can only contain objects that are in both PPMXL and Gaia DR1. Objects that did not make it into the final HSOY are very probably non-stellar objects and failed matches originating in the USNO-B1. However, the inhomogeneous sky coverage of Gaia DR1 (Gaia Collaboration 2016b) most likely also plays a role.

On the other hand, there still is a significant fraction of entries that are probably not related to physical objects in HSOY. The most common form of these are spurious pairs. These may arise from observations that have not been matched in USNO-B, either from different epochs or from different plates. As long as the original USNO-B matched up two observations of the same object for each pair member and the observations had sufficient precision, they form a close, common proper motion pair in PPMXL and are consequently matched to the same Gaia DR1 object. Such objects (and a few other cases where two or more PPMXL objects are matched to the same Gaia DR1 object) are marked with a non-NULL clone flag $(0.7 \%$ of the entire catalogue). The PPMXL catalogue contained about 24.5 million objects with proper motions larger than $150 \mathrm{mas} / \mathrm{yr}$ in the northern hemisphere alone, against an expectation of about $10^{5}$ as discussed in the PPMXL paper. The procedure outlined above brings the number of high-PM objects in HSOY down to 2.5 million on the entire sky $\left(2.5 \times 10^{5}\right.$ in the north). Hence, there are still many spurious high PM objects in HSOY accidentally matching a (real) Gaia DR1 object at J2015. Another reduction of the spurious sources can be effected by matching PPMXL against SuperCOSMOS (Hambly et al. 2001), an independent source extraction from the plate collections underlying USNO-B at $\mathbf{J} 2000$. Where no such match can be found within $3^{\prime \prime}$, HSOY set the no_sc column to 1 . Using only objects with matches in SuperCOSMOS, only 168206 objects with

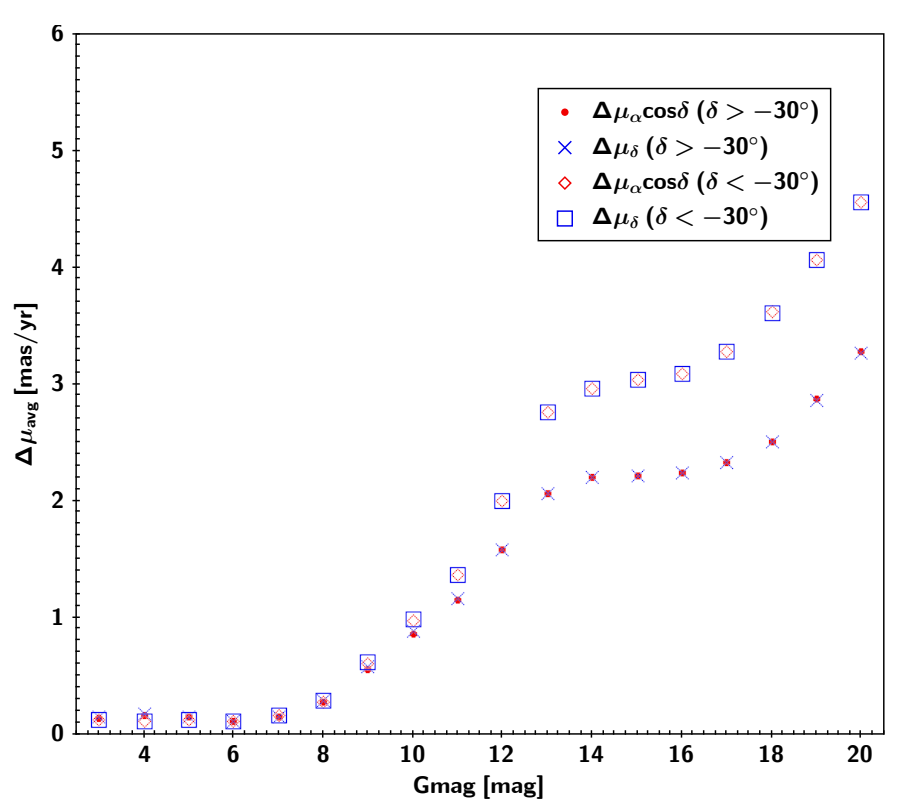

Fig. 2. Mean standard errors in proper motions of the HSOY catalogue north and south of $\delta=-30^{\circ}$. The errors are given in bins of one magnitude centred on full Gaia $G$ magnitudes. The symbols are defined in the legend box within the plot.

$\mu>150$ mas/yr are left in the northern sky within a factor of two of the level to be expected from the Lépine Shara Proper Motion Catalogue (Lépine \& Shara 2005). Over the entire sky, including the very crowded fields in the southern sky, there are about $1.38 \times 10^{6}$ high-PM objects with matches in SuperCOSMOS in HSOY.

Conversely, sometimes more than one Gaia DR1 object is within the match radius of one PPMXL object. While in come cases, this may be due to true binaries already resolved by Gaia, more typically they are due to failed observation matching in the construction of Gaia DR1 and should therefore generally be considered spurious pairs as well. These are marked with a nonNULL comp flag ( $1.5 \%$ of the entire catalogue). In both catalogues, there are a couple of hundred sources that are fainter than $21 \mathrm{mag}$; see Fig. 1. These have to be considered spurious sources.

\subsection{The astrometric precision of HSOY}

For the positions, the overwhelming precision of Gaia DR1 results in mean epochs close to that of Gaia DR1 of 2015.0; the mean epoch of most objects in HSOY is near 2014.8. In HSOY, the positions are given for epoch J2000.0 by applying proper motions. Also, the formal precision of these positions is entirely determined by the precision of the proper motions. These are at maximum 5 mas/yr (see below), therefore the positional rms errors at J2000.0 are well below 0.1 arcsec and are not individually given in the catalogue. Since for the HSOY proper motions, the PPMXL positions are necessary, the vastly higher precision of the Gaia DR1 positions does not dominate as in the case of the mean-epoch positions in the HSOY catalogue. This also means that they reflect some of the systematic errors in the PPMXL, such as the zonal errors present in all ground-based catalogues of similar type (Roeser et al. 2010). This is to be kept in mind when using HSOY proper motions. These systematics are reduced to a certain extent owing to the addition of Gaia DR1, but they do not 

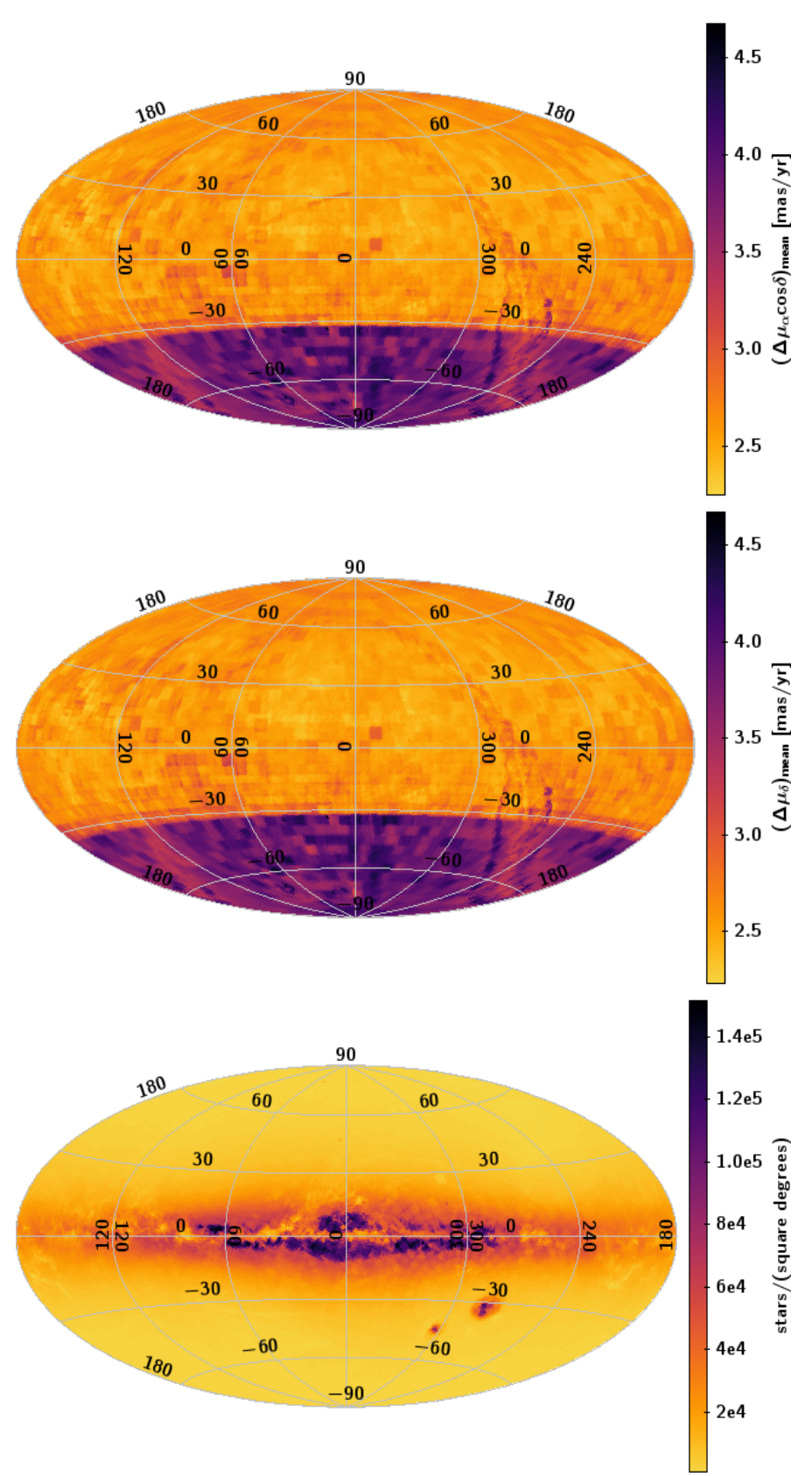

Fig. 3. Upper two panels: global variation of the rms errors in proper motion (upper panel, right ascension, middle panel, and declination) in equatorial coordinates. Darker shades/colours indicate higher errors. Bottom panel: density of HSOY in stars per square degrees over the entire sky in Galactic coordinates highlighting the overall uniformity of the catalogue.

vanish altogether ${ }^{1}$. Given the much smaller positional errors of Gaia DR1, the correlations between the errors in RA and declination in Gaia DR1 can be neglected here. Generally the average formal errors for HSOY proper motions range from $<0.2 \mathrm{mas} / \mathrm{yr}$ for stars brighter than 8 mag up to 4 or 5 mas/yr near the faint magnitude limit; see Fig. 2.

Figure 3 exhibits much larger errors in both proper motion components at declinations south of $-30^{\circ}$; the mean formal errors there are slightly less than double those in the rest of the

\footnotetext{
1 It would in principle be possible to reduce these errors even further by reconstructing the PPMXL itself using Gaia DR1, but this is not worthwhile on the timescale of the useful life of HSOY
}

sky, as shown in Fig. 2. This is inherited from the underlying plate surveys: the first all-sky Schmidt plate surveys started in the northern hemisphere in the 1950s and were extended to the south only much later in the 1970s. Therefore the baseline for proper motions in the southern quarter of the sky is shorter by 20 years with the corresponding consequences for the formal proper motion uncertainties. Apart from this issue the errors in proper motion over the whole sky are remarkably homogeneous because they are just a little higher near the dense areas of the Milky Way. We used the original PPMXL proper motions rather than the possibly more inertial proper motions given by Vickers et al. (2016), since the latter are only available for objects with 2MASS photometry and hence less than half the PPMXL.

HSOY is not a dedicated photometric catalogue, therefore it utilises all photometry that its input catalogues supply. From its PPMXL parent, it retains the photographic magnitudes taken from the USNO-B1 catalogue and the NIR 2MASS values. The Gaia DR1 $G$ magnitude is added to this. Therefore, for more information regarding the quality of the photometry, we refer to the original sources, i.e. Gaia DR1, USNO-B1, and 2MASS.

As for Gaia DR1 itself, the primary access mode to HSOY is the Virtual Observatory protocol TAP ${ }^{2}$. Further access options are discussed online ${ }^{3}$ (Demleitner et al. 2015). Table A.1 shows the data content of HSOY.

\section{The proper motions of M 4 and $M \quad 67$ as science case examples}

In order to demonstrate the increased capabilities of HSOY, especially with respect to earlier, entirely ground-based catalogues, e.g. PPMXL, we present the proper motion distributions in the fields of the globular cluster M 4 (NGC 6121) and the rich, old open cluster M 67 (NGC 2682). These objects are especially instructive, given their large proper motion and high stellar density.

We downloaded a circular field with a radius of $0.5^{\circ}$ for M 4 from both catalogues; this field was found to show both the cluster and field stars best. The field of view is shown in Fig. 4. The resulting vector point diagrams (VPD) are also shown in Fig. 4. A comparison of the VPD made from the PPMXL proper motions (centre panel) with that made from HSOY data clearly shows the dramatic improvement. Although the PPMXL has significantly more stars in this field than HSOY (42 000 versus 30000 ), the M 4 proper motion peak is much more pronounced and the field peak somewhat more pronounced in the latter case.

The open cluster, M 67, one of the oldest of its kind and rather populous, is our second demonstration object. Figure 5 shows the field and vector point diagrams for both PPMXL (centre) and HSOY (right), this time, given the less dense environment, using a field with a radius of $1^{\circ}$. Again the clear improvement is seen by comparing the centre and right panels in Fig. 5 . While the PPMXL only shows hints of the cluster, it clearly stands out in the VPD generated using HSOY.

Both demonstration cases, i.e. M 4 and M 67 highlight the scientific potential of this catalogue. The improvement is clearly shown. There are certainly many other science cases that will profit from the existence of HSOY.

\footnotetext{
2 http://dc.g-vo.org/tap

3 http:///dc.g-vo.org/hsoy
} 

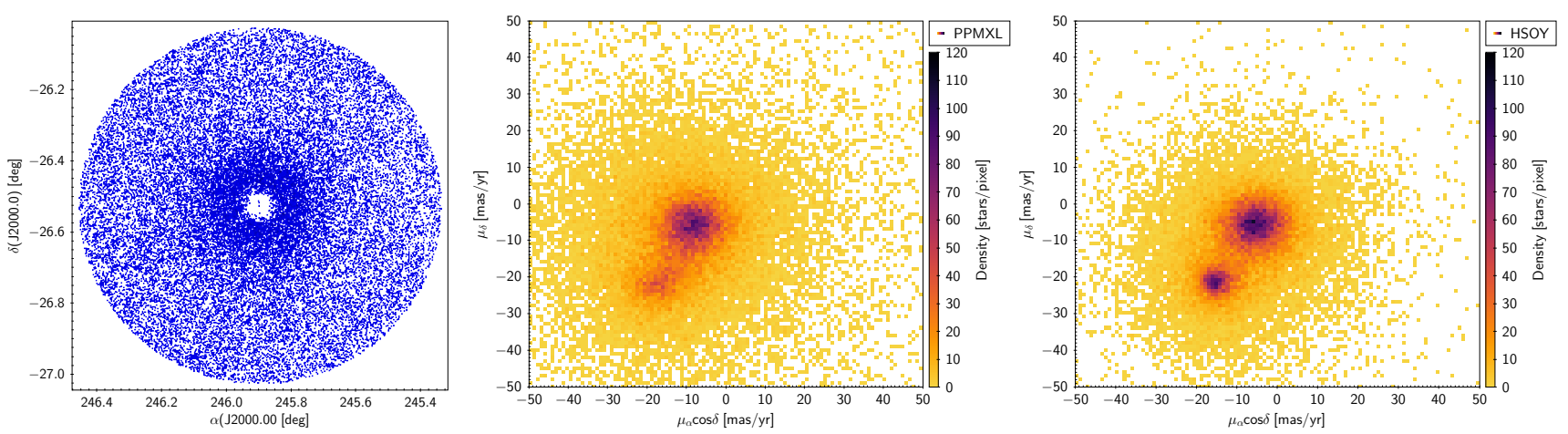

Fig. 4. Left panel: plot of the field with a radius of $30^{\prime}$ taken from the HSOY catalogue centred around M 4 . Centre and right panels: vector point diagrams of the M 4 region with proper motions taken from the PPMXL (centre) and HSOY (right). The hole in the middle of the plot is caused by the strong crowding in the central part of M 4; the few points inside this hole can be considered as spurious, which means they have to be suppressed in any kind of analysis.
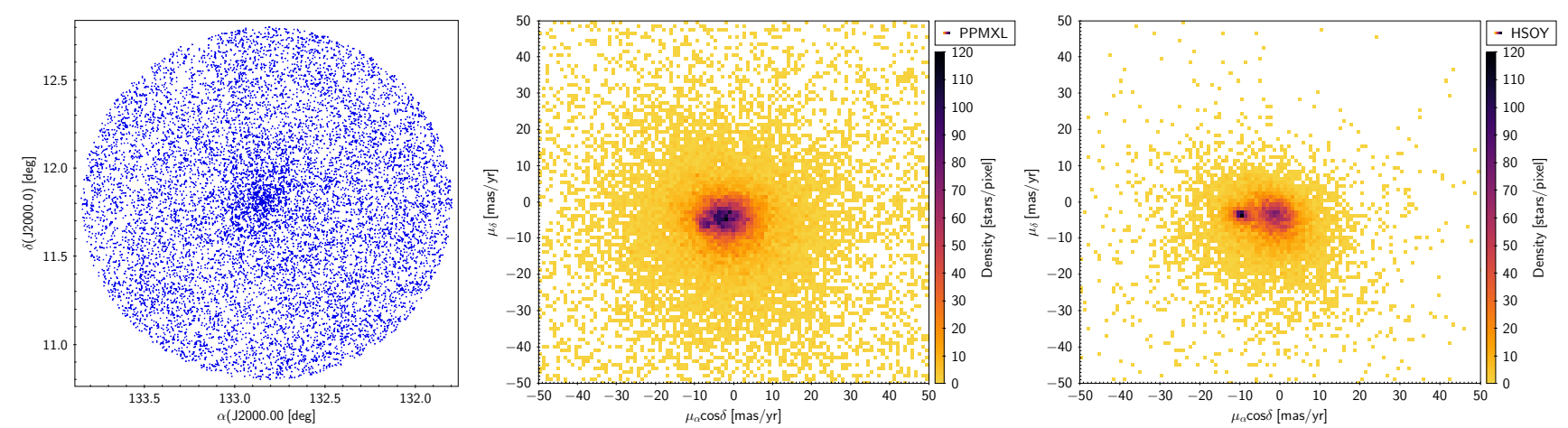

Fig. 5. Left panel: plot of the field with a radius of 1 degrees around M 67 taken from the HSOY catalogue. Centre and right panels: vector point diagrams of the M 67 region with proper motions taken from the PPMXL (centre) and HSOY (right).

\section{Outlook}

In a little more than a year from now, Gaia DR2 will be released and will thus make HSOY obsolete. However, we believe that this catalogue will be put to good use until then. In a way it is the final version of the second-generation ground-based astrometric catalogues, i.e. those developed before or at the beginning of the CCD age, but with old photographic plates as the long timebaseline basis. On the other hand, it presents a bridge to a new generation of ground-based astrometric surveys now based on Gaia data, such as what will come out of LSST. These will reach objects much fainter than either the current catalogues or Gaia and will continue the tradition of space-calibrated ground-based astrometric catalogues.

Acknowledgements. S. Roeser and E. Schilbach were supported by Sonderforschungsbereich SFB 881 "The Milky Way System" (subprojects B5) of the German Research Foundation (DFG). It is a great pleasure to acknowledge Mark Taylor from the Astrophysics Group of the School of Physics at the University of Bristol for his wonderful work on TOPCAT, Tool for OPerations on Catalogues And Tables and STILTS, Starlink Tables Infrastructure Library Tool Set. This research has made use of the resources of CDS, Strasbourg, France. Technical and publication support was provided by GAVO under BMBF grant 05A14VHA.
This work has made use of data from the European Space Agency (ESA) mission Gaia (http://www. cosmos. esa.int/gaia), processed by the Gaia Data Processing and Analysis Consortium (DPAC, http://www . cosmos.esa.int/ web/gaia/dpac/consortium). Funding for the DPAC has been provided by national institutions, in particular the institutions participating in the Gaia Multilateral Agreement.

\section{References}

Demleitner, M., Roeser, S., \& Proft, S. 2015, HSOY Object Catalog, http://dc.zah.uni-heidelberg.de/tableinfo/hsoy.main ESA 1997, ESA SP, 1200

Gaia Collaboration (Prusti, T., et al.) 2016a, A\&A, 595, A1

Gaia Collaboration (Brown, A. G. A., et al.) 2016b, A\&A, 595, A2 Hambly, N. C., MacGillivray, H. T., Read, M. A., et al. 2001, MNRAS, 326, 1279

Høg, E., Fabricius, C., Makarov, V. V., et al. 2000, A\&A, 355, L27

Lépine, S., \& Shara, M. M. 2005, AJ, 129, 1483

Michalik, D., Lindegren, L., \& Hobbs, D. 2015, A\&A, 574, A115

Monet, D. G., Levine, S. E., Canzian, B., et al. 2003, AJ, 125, 984

Roeser, S., Demleitner, M., \& Schilbach, E. 2010, AJ, 139, 2440

Skrutskie, M. F., Cutri, R. M., Stiening, R., et al. 2006, AJ, 131, 1163

Vickers, J. J., Röser, S., \& Grebel, E. K. 2016, AJ, 151, 99 


\section{Appendix A: The data content of the HSOY}

\section{catalogue}

The data content, its units and a short description of every identifier is given in Table A.1.

Table A.1. Content of the HSOY catalogue.

\begin{tabular}{|c|c|c|c|}
\hline & Name & Unit & description \\
\hline 1 & ipix & - & PPMXL object identifier \\
\hline 2 & comp & - & $\begin{array}{l}\text { flag indicating multiple Gaia } \\
\text { matches to one PPMXL object }\end{array}$ \\
\hline 3 & raj2000 & degrees & RA at J2000.0, epoch 2000.0 \\
\hline 4 & de j2000 & degrees & Dec J2000.0, epoch 2000.0 \\
\hline 5 & e_ra & degrees & Mean error: $\alpha \cos \delta$ at mean epoch \\
\hline 6 & $e \_d e$ & degrees & Mean error: $\delta$ at mean epoch \\
\hline 7 & pmra & $\mathrm{deg} / \mathrm{yr}$ & Proper motion in $\alpha \cos \delta$ \\
\hline 8 & pmde & $\mathrm{deg} / \mathrm{yr}$ & Proper motion in $\delta$ \\
\hline 9 & e_pmra & $\mathrm{deg} / \mathrm{yr}$ & Mean error in $\mu_{\alpha} \cos \delta$ \\
\hline 10 & $e_{-} p m d e$ & $\mathrm{deg} / \mathrm{yr}$ & Mean error in $\mu_{\delta}$ \\
\hline 11 & epra & $\mathrm{yr}$ & Mean epoch in RA $(\alpha)$ \\
\hline 12 & epde & yr & Mean epoch in Dec $(\delta)$ \\
\hline 13 & jmag & mag & 2MASS $J$ magnitude \\
\hline 14 & $e_{-} j m a g$ & mag & error of 2MASS $J$ mag. \\
\hline 15 & hmag & mag & 2MASS $H$ magnitude \\
\hline 16 & e_hmag & mag & error of 2 MASS $H$ mag. \\
\hline 17 & kmag & mag & 2MASS $K$ magnitude \\
\hline 18 & e_kmag & mag & error of $2 \mathrm{MASS} K \mathrm{mag}$. \\
\hline 19 & $\bar{b} 1 \mathrm{mag}$ & mag & $B$ mag: USNO-B, 1st epoch \\
\hline 20 & b2mag & mag & $B$ mag: USNO-B, 2nd epoch \\
\hline 21 & $r 1 m a g$ & mag & $R$ mag: USNO-B, 1 st epoch \\
\hline 22 & $r 2 m a g$ & mag & $R$ mag: USNO-B, 2nd epoch \\
\hline 23 & imag & mag & I mag: USNO-B \\
\hline 24 & surveys & - & Origin of USNO-B mags \\
\hline 25 & nobs & - & $\begin{array}{l}\text { total number of astrometric obser- } \\
\text { vations }\left(n_{\mathrm{PPMXL}}+1\right)\end{array}$ \\
\hline 26 & gaiaid & - & Gaia unique source identifier \\
\hline 27 & Gmag & mag & mean Gaia $G$-band magnitude \\
\hline 28 & e_Gmag & mag & estimated error of Gaia $G$-mag \\
\hline 29 & clone & - & $>1$ PPMXL match to Gaia object \\
\hline 30 & no_sc & - & object not in SuperCOSMOS \\
\hline
\end{tabular}

